\section{D) Check for updates}

Cite this: Food Funct., 2021, 12, 7773

\title{
Effects of psyllium seed husk powder, methylcellulose, pregelatinised starch, and cold water swelling starch on the production of gluten free crackers
}

\author{
Yi Ren, (D)*a Bruce R. Linter ${ }^{\mathrm{b}}$ and Tim J. Foster ${ }^{* a}$
}

\begin{abstract}
The production of gluten free crackers is challenging because the formation of a gluten network is required. This study investigated the effects of psyllium seed husk powder (PSY), methylcellulose (MC), pregelatinised starch (PGS), and cold water swelling starch (CWSS) on gluten free crackers made of rice flour. The evaluations of pasting properties, dough rheological properties, textural properties, acoustic emissions, and structures were included in this study. Gluten free cracker doughs were more solid-like compared to wheat doughs based on their frequency dependence shown in the mechanical spectra. However, PGS significantly increased the fluid-like property and shapeability. The addition of MC at a high level significantly modified the pasting profile and a secondary swelling and breakdown might occur. As for the crackers, PSY and PGS crackers had comparable textural properties and sound release to wheat crackers, while CWSS crackers were slightly weaker. However, MC did not improve the textural properties compared to rice crackers because the interaction between the MC molecules was limited at the low water addition level, which limited its functionality in cracker making.
\end{abstract}

Received 22nd December 2020 Accepted 15th June 2021

DOI: $10.1039 / d 0 f o 03377 d$

rsc.li/food-function gas release and drying during baking. ${ }^{3}$ However, gluten free foods need to be developed due to gluten intolerance such as coeliac disease. Celiac disease (CD) is an autoimmune enteropathy, which leads to self-perpetuating damage to the small intestinal mucosa and malabsorption of nutrients. ${ }^{4}$ It can be triggered by the gliadin fraction of proteins in wheat, barley and rye. Currently, a life-long gluten-free diet is the only treatment for the disease. ${ }^{5}$ Because of the important role of gluten in the manufacture of wheat crackers, the production of gluten free crackers is more challenging than the production of gluten free high sugar/short dough biscuits where the formation of gluten network is limited.

Gluten free crackers have been prepared using buckwheat flour, which had comparable sensory quality to wheat crackers. ${ }^{6}$ The levels of antioxidant compounds and antioxidant activity were also found to be improved. ${ }^{6}$ Gluten free crackers were also made from brown rice with the addition of chia seeds to improve the dough texture. ${ }^{7}$ The nutritional quality was improved by adding hemp flour and green tea leaves. ${ }^{7}$ Han $e t ~ a l .{ }^{8}$ produced gluten free crackers made from different pulse flours and both bench-top scale and commercial-scale processing were investigated. Most studies on gluten free crackers focus on nutritional quality and sensory profiles with characterisation of the physical properties of the products. However, investigations on the rheological properties of 
cracker doughs and the structures of crackers are limited compared to the published investigations on gluten free bread.

The addition of various hydrocolloids and dietary fibres in gluten free bread and doughs has been widely investigated and gluten free bread with improved qualities has been produced. ${ }^{9-14}$ Dietary fibres can be either soluble or insoluble. Most of them significantly modify the rheological properties of doughs and influence the structure and texture of food products. ${ }^{10,13-15}$ Additionally, dietary fibres have many health benefits such as improving intestinal peristalsis, eliminating intestinal toxins, helping weight control, and helping to prevent breast cancer, several chronic diseases, type II diabetes and cardiovascular diseases. ${ }^{16-20}$

Starch and modified starch are also widely utilised in the food industry, among which pre-gelatinised starch (PGS) is made from cooked starch and dried using a drum dryer or prepared in an extruder. The degree of gelatinisation of PGS ranges from $44 \%$ to $100 \%$ with the degree of crystallinity ranging from $14 \%$ to $18 \%$ depending on the processing procedures and measuring methods. ${ }^{21}$ Without the starch granular structure, which is broken down during the process, most PGS forms a smooth paste in an aqueous system. PGS is widely used as a thickener in beverages, soups and toppings, a moisture retainer in cakes and a filler in puddings and cheese. ${ }^{21,22}$ It is also widely used in the production of lowmoisture snacks. ${ }^{21,22}$ Being different from PGS, cold-waterswelling starch (CWSS) has intact starch granules. Several methods and conditions have been applied to prepare CWSS including increased temperature, increased pressure, aqueous alcohol, alcohol/alkaline solution, and/or spray-drying with a special nozzle. ${ }^{23-25}$ CWSS made from waxy maize at high temperature in aqueous ethanol was reported showing an amorphous diffraction pattern. ${ }^{26}$ However, the influences on the crystallinity and the overall starch granules depend on the types of starch and the process applied. CWSS is applied as a thickener, stabiliser, and fat replacer to improve the texture and mouth-feel and is used to make gum candies. ${ }^{21,27,28}$ Although CWSS can also be classified as pre-gelatinised starch, CWSS and PGS are used in this study to identify these two different types of modified starch.

This study investigated the applications of methylcellulose (MC), powder of psyllium (Plantago ovata) seed husk (PSY), PGS and CWSS in the production of gluten free crackers. The influences of different gums and modified starch on the pasting properties of flour blends, dough rheological properties, textural properties of crackers, and structures of doughs and crackers were evaluated and compared. Additionally, acoustic emission was simultaneously recorded with mechanical measurements.

\section{Materials and methods}

\subsection{Materials}

Rice flour containing $11.08 \%$ moisture, $7.23 \%$ protein, $0.42 \%$ ash, and $2.8 \%$ lipid with an amylose content of $28.79 \%$ as measured in our previous study ${ }^{29}$ was purchased from Doves Farm. Dr Oetker Gluten Free Baking Powder, caster sugar (Sainsbury's, UK), salt (Sainsbury's, UK), sunflower oil (Sainsbury's, UK), and unsalted butter (Morrisons, UK) were purchased from local supermarkets. Psyllium husk powder (Vitacel®) was kindly donated by the J. Rettenmaier \& Söhne Group (JRS, Rosenberg, Germany). Methylcellulose (Methocel® A4M) was supplied by The Dow Chemical Company (Bomlitz, Germany). Pregelatinised waxy maize starch (Merigel®) was supplied by Tate \& Lyle (UK). Cold water swelling waxy maize starch (Ultra-Tex® 4) was kindly donated by Ingredion (UK). Nile red (Sigma-Aldrich), fluorescein isothiocyanate (FITC, Acros Organics, New Jersey, USA), methyl blue (Sigma-Aldrich, UK), fast green (Sigma-Aldrich) and rhodamine B (SigmaAldrich) were of analytical grade.

The water absorption capacity of rice flour was 133\% (118\% after being corrected for the moisture content of flour), which was determined using time-domain nuclear magnetic resonance (NMR). Flour was hydrated overnight before measurements. The Carr-Purcell-Meiboom-Gill (CPMG) sequence ${ }^{30}$ was applied to obtain the transverse relaxation curves using an R4 Benchtop NMR System (Advanced Magnetic Resonance Ltd, Abingdon, UK) at $20^{\circ} \mathrm{C}$, which was controlled using a thermal controller (Advanced Magnetic Resonance Ltd). The initial $90^{\circ}$ pulse for all the sequences was approximately $2.6 \mu$ s and the signals were recorded after $10 \mu \mathrm{s}$ (dead time). There were 4096 echoes for the following $180^{\circ}$-pulses with $64 \mu \mathrm{s}$ (TAU) between every two pulses. The results were obtained averaging 32 scans with a recycle delay of $10 \mathrm{~s}$. The distributed exponential using the Lexus fixed method was applied to calculate the T2 distribution spectra. The water absorption capacity was determined at the water addition level when the T2 peak at approximately $700 \mathrm{~ms}$ for the unabsorbed bulk water was absent.

\subsection{Formulation and cracker preparation}

The formulation of gluten free crackers was optimised singly for rice, MC, PSY, PGS, CWSS and wheat crackers by trial and error and is shown in Table 1. For rice, MC, and PSY crackers, the water addition levels were determined as required to form the doughs under the applied mixing conditions. The water addition levels for wheat, PGS and CWSS crackers were determined by trial and error and kept constant. Doughs were mixed using a Kenwood stand mixer (UK) equipped with a Chef $\mathrm{K}$ beater. To prepare the cracker doughs, all powdered ingredients were mixed and combined with butter (stored at $10{ }^{\circ} \mathrm{C}$ ) thoroughly. Sunflower oil and water were mixed and gradually added over $1 \mathrm{~min}$ at speed 2 . The dough was then mixed at speed 4 for $2 \mathrm{~min}$. After resting at $5{ }^{\circ} \mathrm{C}$ for $10 \mathrm{~min}$, the doughs were laminated to 16 layers and sheeted to $1.6 \mathrm{~mm}$ thickness $(2.6 \mathrm{~mm}$ for rice and MC cracker doughs because they were very fragile and difficult to handle). The dough sheets were cut into $36 \times 36 \mathrm{~mm}$ squares on a piece of baking parchment, transferred to a preheated baking tray and baked in a fan oven (Hotpoint, UK) at $250{ }^{\circ} \mathrm{C}$ for 6 min and $105{ }^{\circ} \mathrm{C}$ for $10 \mathrm{~min}$. The crackers were cooled at room temperature for $1 \mathrm{~h}$ and stored in polyethylene bags for further analysis. 
Table 1 Addition levels of the ingredients in the cracker formulation

\begin{tabular}{|c|c|c|c|c|c|c|}
\hline & $\begin{array}{l}\text { Rice crackers } \\
\text { (negative control) }\end{array}$ & $\begin{array}{l}\text { Wheat crackers } \\
\text { (positive control) }\end{array}$ & $\begin{array}{l}\text { MC } \\
\text { crackers }\end{array}$ & $\begin{array}{l}\text { PSY } \\
\text { crackers }\end{array}$ & $\begin{array}{l}\text { PGS } \\
\text { crackers }\end{array}$ & $\begin{array}{l}\text { CWSS } \\
\text { crackers }\end{array}$ \\
\hline Rice flour & 100 & 0 & 100 & 100 & 80 & 70 \\
\hline Wheat flour & 0 & 100 & 0 & 0 & 0 & 0 \\
\hline Baking powder & 5 & 5 & 5 & 5 & 5 & 5 \\
\hline Sugar & 5 & 5 & 5 & 5 & 5 & 5 \\
\hline Salt & 1 & 1 & 1 & 1 & 1 & 1 \\
\hline Butter & 30 & 30 & 30 & 30 & 30 & 30 \\
\hline Oil & 10 & 10 & 10 & 10 & 10 & 10 \\
\hline Water & 52 & 50 & 71 & 71 & 50 & 50 \\
\hline MC & 0 & 0 & 4 & 0 & 0 & 0 \\
\hline PSY & 0 & 0 & 0 & 4 & 0 & 0 \\
\hline PGS & 0 & 0 & 0 & 0 & 20 & 0 \\
\hline CWSS & 0 & 0 & 0 & 0 & 0 & 30 \\
\hline
\end{tabular}

\subsection{Pasting properties of the flour blends}

The influences of the addition of MC, PSY, PGS and CWSS on the pasting profiles of the flour blends were evaluated using a Rapid Visco Analyser (RVA) (Newport Scientific Pty Ltd, Warriewood, New South Wales, Australia) and the temperature control was assisted by a water bath (Thermo Scientific C10, Karlsruhe, Germany). To prepare the hydrocolloid blends, $0.1 \mathrm{~g}$ of MC or PSY was mixed with $2.5 \mathrm{~g}$ of rice flour and dispersed in $24 \mathrm{~g}$ of reverse osmosis (RO) water in an aluminium canister. The PGS blend and CWSS blend were prepared by substituting $20 \%$ of the rice flour with starch and $3.125 \mathrm{~g}$ of the flour blends were added into $21.875 \mathrm{~g}$ of RO water. During the measurements, the flour or flour blends were dispersed thoroughly at a high shear rate $(960 \mathrm{rpm})$ for $60 \mathrm{~s}$, followed by a low rate shearing $(160 \mathrm{rpm})$ for another 60 seconds at $25{ }^{\circ} \mathrm{C}$. Keeping the shear rate constant at $160 \mathrm{rpm}$, the temperature profile started with an increase from $25{ }^{\circ} \mathrm{C}$ to $95{ }^{\circ} \mathrm{C}$ in $350 \mathrm{~s}$, maintained at $95{ }^{\circ} \mathrm{C}$ for $150 \mathrm{~s}$, and decreased back to $25^{\circ} \mathrm{C}$ in $350 \mathrm{~s}$. The measurements were repeated three times for each sample.

\subsection{Dough rheology}

Cracker doughs were prepared as described in section 2.2 without the addition of baking powder. The doughs were sheeted to $2.5 \mathrm{~mm}$ and loaded on an MRC 301 rheometer (Anton Paar, Austria) equipped with a Peltier system controlling the temperature. The measurements were performed using a serrated parallel plate geometry (PP25/P2-SN15766, Anton Paar) and the measuring gap was maintained at $2 \mathrm{~mm}$. After being loaded, the extra doughs were trimmed by a spatula and the edge was covered with low viscosity mineral oil (Sigma, USA) to prevent drying. After resting at $20{ }^{\circ} \mathrm{C}$ for $500 \mathrm{~s}$, the doughs were subjected to oscillatory shear tests with a logarithmic decrease of angular frequency at a constant shear strain of $0.02 \%$, which was within the linear viscoelastic regions for all the doughs. The measurements were performed in triplicate.

\subsection{Cracker evaluation}

The thickness was measured using a vernier caliper by averaging the thickness measured at the different positions ( 4 to 8 positions) on a piece of cracker. The water activity of crackers (powdered using a coffee grinder) was measured using an Aqua Lab water activity meter (Aqua Lab, 3TE, Decagon, Pullman, WA, USA). The moisture contents of the crackers were measured by drying at $105^{\circ} \mathrm{C}$.

The crackers were further evaluated by three-point bending tests on a TA-XT plus texture analyser (Stable Micro Systems, Surrey, UK) equipped with $5 \mathrm{~kg}$ loading cell and the sound emitted during testing was recorded using an Acoustic Envelope Detector (Stable Micro Systems). The distance between the two lower supporting points was $18 \mathrm{~mm}$. The upper-middle point descended at a speed of $2 \mathrm{~mm} \mathrm{~s}^{-1}$ to a distance of $5 \mathrm{~mm}$ after the surface of the crackers was detected. The trigger force was $5 \mathrm{~g}$. The Acoustic Envelope Detector was equipped with a microphone located close to the fracture positions (about $2 \mathrm{~cm}$ ). The microphone was calibrated to 94 and $114 \mathrm{~dB}$ SPL using a sound level calibrator. The gain was set to four. Sixteen crackers were evaluated for each formulation. The maximum force was reported as hardness of the crackers and the travelling distance of the probe to the first force peak was reported as fracturability. The intensity of the emitted sound was described by the maximum sound peak, the average peak decreases, and the number of sound peaks. A decrease in the sound pressure level to $8.5 \mathrm{~dB}$ was set as the threshold to identify the peaks and calculate the average peak decreases.

\subsection{Confocal laser scanning microscopy}

The microstructure of the cracker doughs was observed using a Zeiss LSM880 confocal laser scanning microscope (Carl Zeiss Microscopy GmbH, Jena, Germany). Stock solutions of the stains were prepared, including $1 \mathrm{~g} \mathrm{~L}^{-1}$ Nile red in acetone, $1 \mathrm{~g}$ $\mathrm{L}^{-1}$ FITC in acetone, $1 \mathrm{~g} \mathrm{~L}^{-1}$ methyl blue in water, $1 \mathrm{~g} \mathrm{~L}^{-1}$ rhodamine $\mathrm{B}$ in water and $1 \mathrm{~g} \mathrm{~L}^{-1}$ fast green in water. The stock solutions were diluted and used to make the working solutions containing different stains specific for different doughs with a final concentration of $0.1 \mathrm{~g} \mathrm{~L}^{-1}$ for each stain. The working solutions were added to the doughs replacing the water in the formulation. A working solution containing fast green and rhodamine B was used to stain gluten and starch/lipid, respectively, in the wheat doughs. FITC and Nile red were used to stain starch and lipid, respectively, in the rice doughs, PGS 
doughs and CWSS doughs. FITC, Nile red and methyl blue stained starch, lipid and PSY in the PSY cracker doughs. Fast green was excited at $633 \mathrm{~nm}$ and the signal was collected at 641 to $710 \mathrm{~nm}$. Rhodamine B was excited at $488 \mathrm{~nm}$ and the signal was collected at 545 to $590 \mathrm{~nm}$. FITC and Nile red were both excited at $488 \mathrm{~nm}$, while the signal collection wavelengths were 510-540 $\mathrm{nm}$ and 570-600 $\mathrm{nm}$, respectively. Images were acquired at $20 \times$ magnification using an EC Plan Neofluar@ objective.

\subsection{Scanning electron microscopy}

The powdered ingredients including PSY, MC, PGS, and CWSS and the crackers were evaluated using scanning electron microscopy (JEOL JSM-6060LV, JEOL (UK) Ltd, Welwyn Garden City, UK). The powdered ingredients were mounted onto aluminium stubs using carbon tapes. The crackers were broken and a piece of cracker was mounted onto the aluminium stub using carbon tapes with the exposed surface facing up. The powdered ingredients and cracker pieces were coated with gold using a Leica EM SCD005 Sputter Coater at an argon current rate of $27 \mathrm{~mA}$ for $3 \mathrm{~min}$. The images were acquired under high vacuum conditions at $10 \mathrm{kV}$.

\subsection{Statistical analysis}

Representative curves and images were selected from the replicates for each sample and are shown in the figures, while the means and standard deviations were calculated and are shown in the tables. Additionally, the results were analysed and compared using one-way analysis of variance (ANOVA) with Turkey's test using IBM SPSS Statistics (version 26, IBM Corp., Armonk, NY, USA) at a significance level of $p<0.05$.

\section{Results and discussion}

\subsection{Microstructures of the raw ingredient powders}

The microstructures of rice flour, PSY, MC, PGS, and CWSS were scanned using a scanning electron microscope and the microscopic images are shown in Fig. 1. Fig. 1a shows that rice flour contains both small and big flour particulates. Rice starch exists as compound granules which consist of small individual starch granules ${ }^{31}$ and Fig. $1 \mathrm{~b}$ shows that the granules are enclosed in proteinaceous materials and other cell contents. ${ }^{32}$ Fig. 1c and d show PSY at different magnifications. It can be seen in Fig. 1d that PSY particles show a lamellar structure, which is in agreement with the results of the study by Mariotti et $a l^{33}$ and the observation is dependent on the positions of observation. The functional polymer of PSY, which is heteroxylan, is likely to be expelled from this lamellar structure when hydrated in water and swells into 'weak-gel' particles. ${ }^{34}$ Fig. 1e and $\mathrm{f}$ show that the MC powder is composed of fibrous particles. In the production of methylcellulose, native cellulose is only activated in heated alkaline solutions without being fully dissolved; therefore, the product is fibrous like native cellulose.$^{35,36}$ The fibrous structure of the MC powder could be a clue to the distribution of the MC molecules in cracker doughs and crackers as they might not be fully hydrated due to the low moisture content in the formulation. Fig. $1 \mathrm{~g}$ and $\mathrm{h}$ show the microstructure of PGS and Fig. 1i and $\mathrm{j}$ show that of CWSS. As expected, CWSS shows individual starch granules which are not observed for PGS. ${ }^{37,38}$ These modified starch granules of CWSS absorb water and swell at room temperature.

\subsection{Pasting properties}

The influences of the addition of PSY and MC and the replacement by PGS and CWSS on the pasting properties of the flour blends were evaluated using RVA 2. As shown in Fig. 2a, the addition of PSY significantly increased the overall viscosity of the paste. However, the MC flour blend showed an atypical pasting profile with three viscosity peaks possibly due to the high addition level of MC which led to high viscosity. The decrease of pasting temperature with the addition of MC and PSY was observed, which was also reported in one of our previous studies due to water and volume competition between starch and the added hydrocolloids. ${ }^{12}$ Although the addition level of MC in the current study was higher than our previous study, the pasting temperature was decreased to a similar value of $70^{\circ} \mathrm{C}$. The pasting temperature was also less influenced by the molecular weight of $\mathrm{MC}$ and different types of $\mathrm{MC}$ or hydroxypropylmethylcellulose. ${ }^{39}$ Therefore, it can be speculated that the decreased pasting temperature is predominantly controlled by the swelling behaviour of starch granules. It is known that MC gelation occurs at 50 to $55^{\circ} \mathrm{C}$ and it forms a rigid gel at high temperature. Therefore, the system of the MC/starch mixture under RVA conditions (high temperature with shearing) can be described as a dispersion of MC gel particles and swollen starch granules. ${ }^{40}$ According to the Krieger-Dougherty model, ${ }^{41}$ viscosity significantly increases when the volume fraction of a suspension reaches the packing fraction, which explains the significant increase in viscosity after the pasting temperature due to the swelling of starch granules. Because the MC particles occupied a certain volume fraction, a lower swelling degree of starch granules could lead to a volume fraction which triggers a significant viscosity increase at a temperature that is identified as pasting temperature. With a lower addition level of MC, viscosity developed a shoulder after this pasting temperature before the significant increase caused by the temperature-trigged, significant swelling of the starch granules, ${ }^{12}$ which was also observed from the PSY blend (Fig. 2a). In a typical pasting profile of starch or flour, the starch granules are closely packed at peak viscosity and the following breakdown is accompanied by a viscosity decrease. Following the initial viscosity increase, the starch granules keep swelling and growing in size, which increases their volume fraction and leads to an increase in viscosity. The increased viscosity causes a higher shear force applied to the starch granules. At the same time, the granules lose their rigidity during swelling due to hydration, melting of the helical conformation, and loss of crystallinity. Therefore, the granules eventually cannot resist the increased shear force and start to lose the granule integrity, which is observed as the decrease in viscosity and identified as the breakdown stage. Hence, the packing at the viscosity peak and breakdown of starch granules can be influenced by the balance 

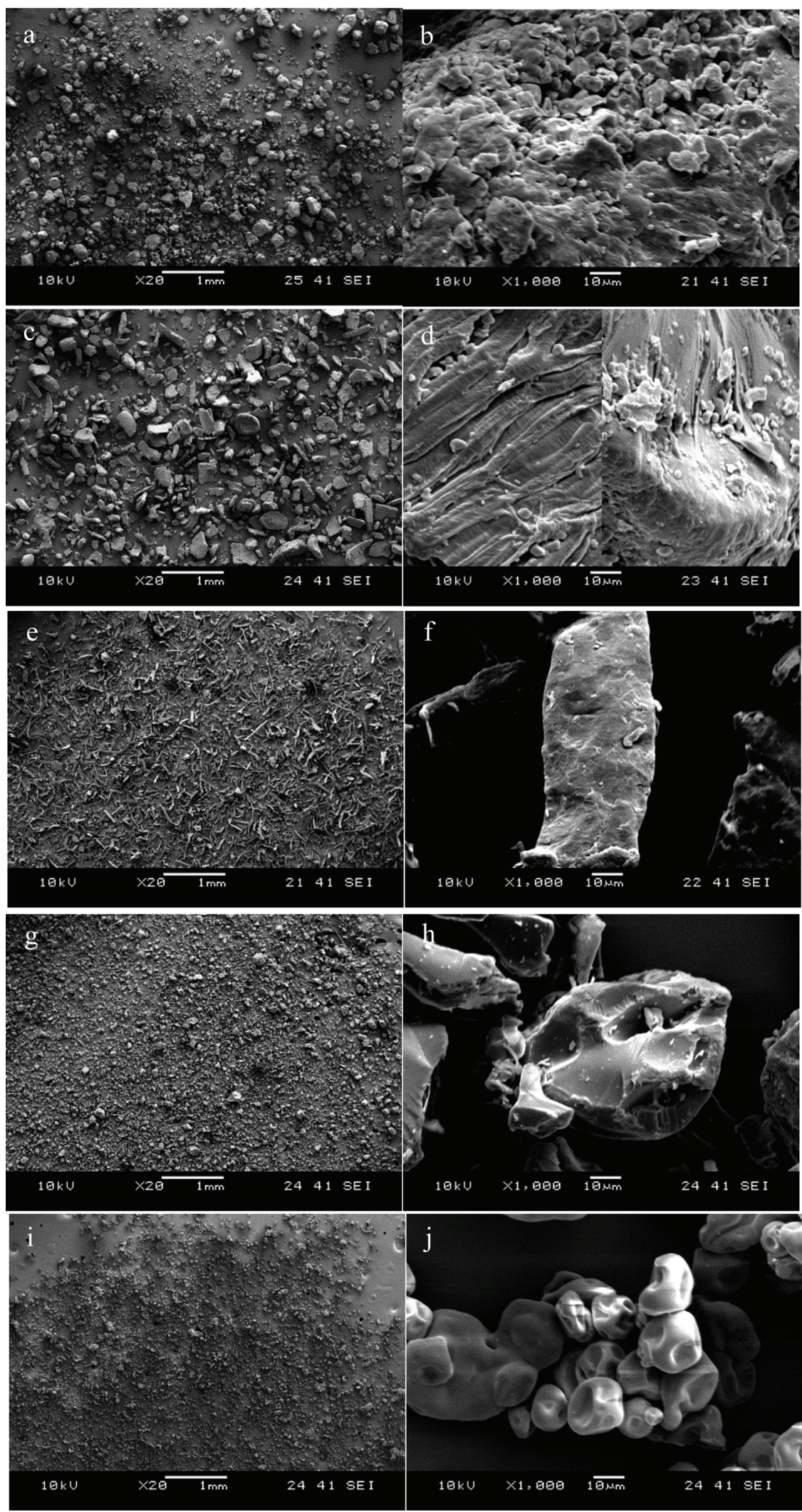

Fig. 1 Scanning electron microscopy images of rice flour ( $a$ and b), PSY powder ( $c$ and d), MC (e and f), PGS ( $g$ and $h$ ) and CWSS ( $i$ and j) used in the gluten free cracker formulation. 
a

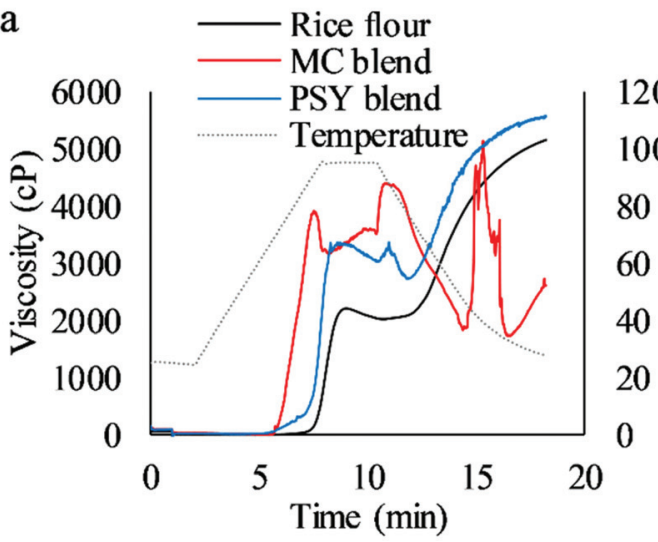

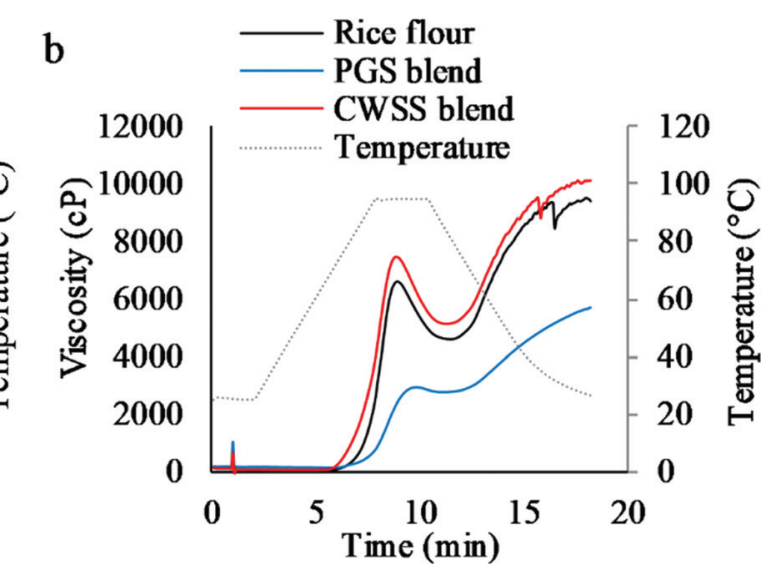

Fig. 2 Pasting profiles of (a) MC blend and PSY blend compared with rice flour (control), where $0.1 \mathrm{~g}$ of MC or PSY was mixed with $2.5 \mathrm{~g}$ of rice flour in $24 \mathrm{~g}$ of water, and (b) PGS blend and CWSS blend compared with rice flour (control), where $3.125 \mathrm{~g}$ of rice flour or flour blends were added into $21.875 \mathrm{~g}$ of water. The flour blends were prepared by $20 \%$ replacement of rice flour by PGS or CWSS. The pasting profiles are shown as representative curves from the experiments run in triplicate.

between the shear force applied on the starch granules due to the high viscosity at high volume fractions and starch granule rigidity. In the case of the MC blend in this study, the significant increase in viscosity due to the MC gel particles caused a higher shear force on the starch granules and they broke down earlier compared to the flour only sample, which was shown as a lower temperature for the peak viscosity (Fig. 2a). As the starch granules in the MC blend broke down earlier, there might be a secondary swelling of the granules and broken granule fragments, which was shown as the slight increase in viscosity from $8 \mathrm{~min}$ to $10 \mathrm{~min}$. However, this small increase was not reproducible and a slight decrease of viscosity was sometimes observed (data not shown) due to the following breakdown instead of swelling. For both MC and PSY blends, the obvious viscosity increase led to a second peak occurring at a slightly earlier time than the end of the breakdown stage of the flour only sample. It is possible because of the continuous granule swelling, the formation of granule ghosts ${ }^{42}$ and the cessation of granule breakdown, and the increased viscosity led to the secondary breakdown after the second peak. It is proposed in addition to what has been proposed in a separate study from our group that the second peak of the PSY blend is due to a balance between the interaction with amylose (James M. Cowley, personal communication), the formation of weak PSY gel particles, PSY particle interactions, and PSY particle breakdown. ${ }^{12}$ Compared to the rice flour and PSY blends, MC significantly decreased retrogradation, which was seen as low viscosity during cooling. An irreproducible peak appeared during this stage because of the disruption of the structure formed by the alignment and association of amylose molecules.

As shown in Fig. 2b, the replacement by CWSS slightly increased the overall viscosity of the pasting profiles compared to the flour only sample. However, the replacement by PGS significantly decreased the overall viscosity due to the absence of the granular structure. The low viscosity was also observed in other studies when the starch granules were disrupted to a high degree during preparation. ${ }^{43}$ Additionally, PGS is soluble in cold water and quickly absorbs water. Therefore, it is likely that PGS was quickly hydrated in cold water and formed a barrier outside the rice flour particulates, which restrained the hydration and swelling of the native rice starch and further decreased the viscosity.

\subsection{The microstructure of the cracker doughs}

The microstructures of the cracker doughs are shown in Fig. 3. As shown in Fig. 3a, the strands of the gluten network were seen in the wheat cracker doughs. The wheat starch granules were shown as dark circles, which indicates that the stain did not penetrate the granules. It suggests that the granules were not fully stained as the hydration of starch was highly limited in the wheat cracker doughs. The rice starch in the rice cracker doughs and PSY cracker doughs (Fig. 3b and c), in contrast, was fully stained in green, which indicates that they were more hydrated. It was possibly due to the higher water addition level, smaller granule size of the rice starch, and the difference in water-binding abilities between gluten and PSY. Although PSY did not form a network like gluten, it formed a matrix which holds the flour particles, starch granules, and other insoluble ingredients together and allows the formation of a cohesive dough. In the PGS and CWSS doughs, both rice starch from rice flour and the added PGS or CWSS were stained in green. Although the starch cannot be distinguished in colour, they were different in shape, size, and distribution. As shown in Fig. 3d, PGS formed a relatively continuous matrix in which the rice flour and starch were embedded. In contrast, swollen starch granules from CWSS can be observed in the CWSS dough (Fig. 3e). Being different from the PSY and rice doughs, the rice starch, especially the starch granules within the flour particles, in the PGS and CWSS doughs was less hydrated (less stained and showed lower colour intensity). It was because of the lower water addtiion levels in the formulation which reduced the hydration of rice flour. Additionally, 

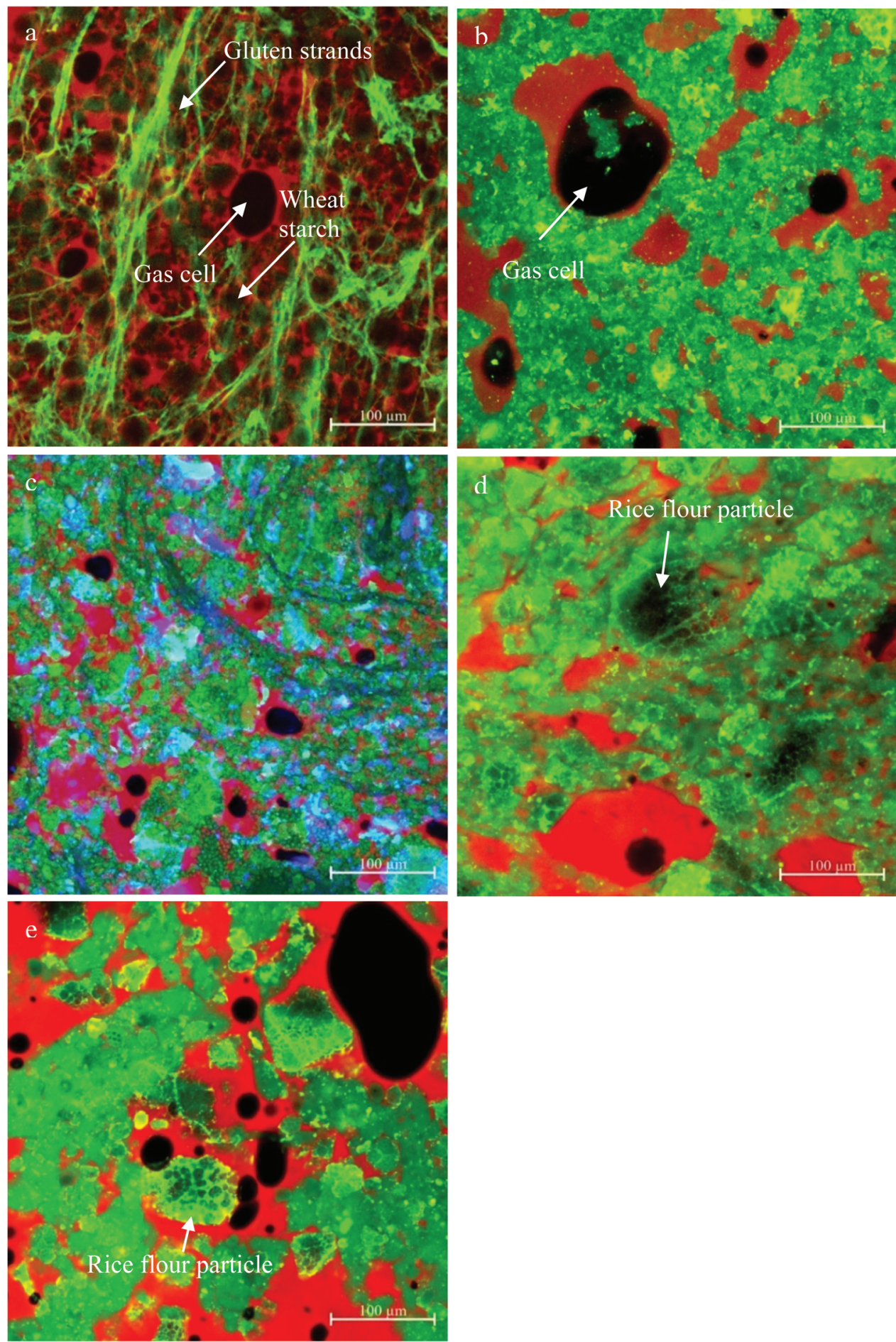

Fig. 3 Confocal laser scanning microscopy images of the wheat cracker doughs (a), rice cracker doughs (b), PSY cracker doughs (c), PGS cracker doughs (d) and CWSS cracker doughs (e). Gluten and starch/lipid in the wheat doughs were stained using fast green and rhodamine B, and are shown in green and red, respectively. In the rice, PSY, PGS and CWSS doughs, starch and lipid were stained using FITC and Nile red, and are shown in green and red, respectively. PSY in the PSY cracker doughs was stained using methyl blue, and is shown in blue.

PGS and CWSS have high water-binding abilities. The additions of PGS and CWSS at high levels further restrianed the hydration of rice flour by competing for water. Lipid, which was stained in red, was distributed in the cracker doughs and accumulated surrounding the gas cells.

\subsection{Rheological properties of the cracker doughs}

The rheological properties of the cracker doughs were measured using oscillatory shear tests. The amplitude sweep spectra are shown in Fig. 4a. Both wheat cracker doughs and 

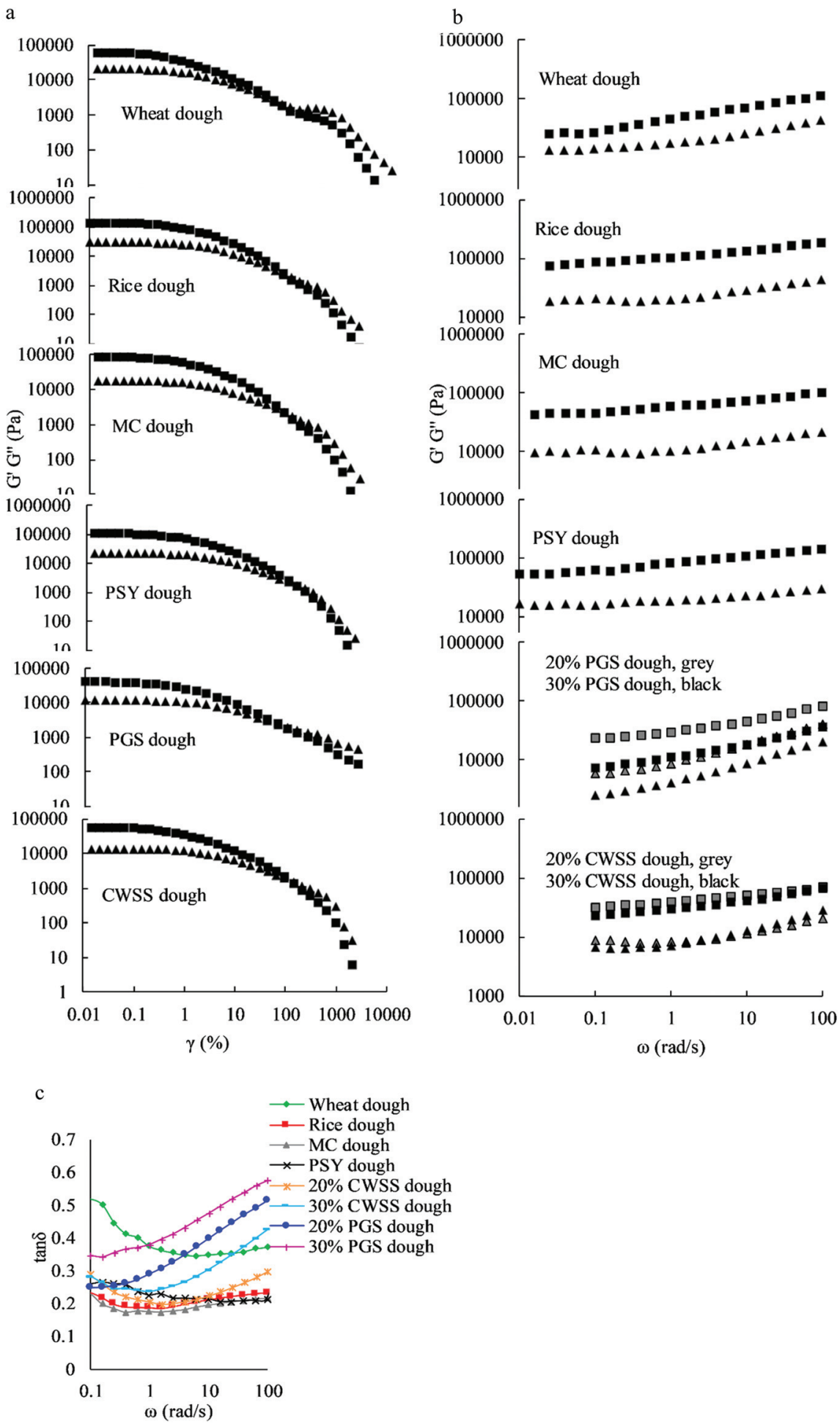

Fig. 4 Storage and loss moduli ( $G$ ' square and $G^{\prime \prime}$ triangle) measured in the strain amplitude sweep tests (a), the mechanical spectra (b), and tan $\delta$ measured in the frequency sweep tests (c) of wheat and all gluten free cracker doughs.

gluten free cracker doughs had similar lengths of linear viscoelastic regions of about $0.1 \%$. As complex concentrated suspensions, they had very short linear viscoelastic regions compared to the polymer solutions and melts which usually have LVE regions higher than $10 \%{ }^{44}$ and gels. The gradual decrease of the moduli with the increase of shear strain outside the LVE region could be due to the breakdown and reformation of the weak network of the rheological units that are composed of flour particles, starch granules and other soluble and insoluble flour components, which weakly interact with the neighbour- 
ing rheological units to different levels. It was evidenced that starch predominantly contributes to the non-linearity of wheat doughs. ${ }^{45}$ However, it is noticed that wheat doughs showed a secondary $G^{\prime}$ and $G^{\prime \prime}$ plateau at high strain (between 100 and $1000 \%$ ), which might be attributed to the gluten network, although the evaluation at large stain must be careful and wall slips need to be taken into consideration.

The mechanical spectra of the cracker doughs are shown in Fig. 4b. As expected, all doughs had higher $G^{\prime}$ than $G^{\prime \prime}$ with $\tan \delta$ lower than 0.6 (Fig. 4c), suggesting solid-like properties. As shown in Fig. 4b, the storage moduli of the gluten free cracker doughs tended to be less dependent on the angular frequency (with $\log G^{\prime}$ versus $\log \omega$ of $0.11,0.11,0.13,0.11,0.14$, 0.18 , and 0.23 for the rice doughs, MC doughs, PSY doughs, 20\% CWSS doughs, 30\% CWSS doughs, 20\% PGS doughs, and $30 \%$ PGS doughs, respectively) than those of the wheat doughs $\left(\log G^{\prime}\right.$ versus $\left.\log \omega: 0.21\right)$ as a result of close interactions between the packed rigid flour particles in the absence of the gluten network. Compared to the rice doughs, the addition of CWSS and, especially, PGS significantly increased the frequency dependence, while the PSY and MC doughs remained less influenced. As shown in Fig. 4c, the lower $\tan \delta$ of the rice, PSY and MC doughs than the wheat doughs suggests that they were more solid-like. However, the addition of CWSS and, especially, PGS significantly increased $\tan \delta$ at high angular frequencies, which were even higher than those of the wheat doughs. It indicates that PGS and CWSS doughs were more fluid-like at shorter time scales. This was consistent with the observation during dough handling that PGS doughs were more shapeable.

\subsection{Cracker properties}

The physical properties of the gluten free crackers are shown in Table 2. As the dough sheets were cut to a dimension of 36 $\times 36 \mathrm{~mm}$, it can be seen that both wheat crackers and all gluten free crackers had lengths shorter than $36 \mathrm{~mm}$, which suggests that they all contracted in the lateral direction during baking. The shrinkage of the wheat crackers was partially due to the elastic properties of the gluten network which caused shrinkage after cutting. It was also due to the elastic shrinkage of the gluten network which displays hardening behaviour above its glass transition temperature. ${ }^{46}$ The shrinkage of the crackers was also caused by water loss under the temperature gradient and moisture gradient during baking. Although the difference in lengths between the MC, CWSS and PSY crackers was insignificant, the PSY crackers contracted to the same length as the wheat crackers. It suggests that heteroxylan from PSY possibly manifested a similar elastic shrinkage behaviour to gluten in the wheat crackers during baking. In contrast, the rice crackers and PGS crackers contracted less. It is possibly because they were predominantly plasticised by water and hydrated PGS, which tended to spread during baking, while the shrinkage due to water loss led to the final shrinkage.

The wheat crackers also featured a higher thickness, which increased by $156 \%$ compared to the thickness of the dough sheets $(1.6 \mathrm{~mm})$. Among the five gluten free crackers, the PGS crackers had the highest thickness, which increased to $2.77 \mathrm{~mm}$ by $73 \%$, followed by the CWSS crackers and PSY crackers. However, the rice and MC crackers showed lower increases in thickness.

The PSY crackers had a relatively high moisture content but low water activity. The increase in the moisture content of the crackers and biscuits when gums were added was also observed previously, ${ }^{47}$ which is assigned to the water retention ability of gums. The high water retention ability of PSY also explains the low water activity of the crackers. Additionally, a porous structure is expected to increase the water loss during baking, but increase the water absorption during storage.

\subsection{Microstructure of the crackers}

The microstructures of the wheat crackers, PSY crackers, PGS crackers and CWSS crackers are shown in Fig. 6. A laminar and plate-like structure was observed in the wheat crackers (Fig. 5a and b). It indicates that the wheat doughs were successfully laminated and the wheat crackers maintained the structure during the gas cell growth, cell ruptures and gas release during baking. Starch and other components existed as intact granules and particles embedded in the gluten matrix. It was also reported by Kulp et al. ${ }^{48}$ that starch in cookie doughs and end products does not swell, gelatinise, and form a continuous network which, however, is usually observed in high moisture products. However, the PSY, PGS, and CWSS crackers did not show an obvious laminar or plate-like structure compared to the wheat crackers. As shown in Fig. $5 \mathrm{c}$ and d, the small rice starch granules were embedded in a continuous matrix in the PSY crackers. The PSY powder hydrated into a gel-like soft particle in water with an insoluble and unhydrated core rich in cellulose. ${ }^{34}$ The PSY core was also seen in the matrix. The con-

Table 2 Physical properties of the wheat and gluten free crackers

\begin{tabular}{|c|c|c|c|c|c|}
\hline & Length (mm) & Thickness (mm) & Thickness increase (\%) & Moisture content (\%) & Water activity \\
\hline Wheat & $32.13 \pm 1.12^{c}$ & $4.10 \pm 0.09^{c}$ & $156.09 \pm 5.74^{\mathrm{b}}$ & $3.19 \pm 0.00^{b c}$ & $0.586 \pm 0.010^{c}$ \\
\hline Rice $^{a}$ & $34.70 \pm 0.57^{\mathrm{ab}}$ & $2.94 \pm 0.14^{\mathrm{a}}$ & $13.08 \pm 5.38^{\mathrm{a}}$ & $4.08 \pm 0.05^{\mathrm{ab}}$ & $0.394 \pm 0.007^{\mathrm{a}}$ \\
\hline $\mathrm{MC}^{a}$ & $33.13 \pm 1.28^{c}$ & $2.71 \pm 0.19^{\mathrm{ab}}$ & $4.04 \pm 7.34^{\mathrm{a}}$ & $2.46 \pm 0.43^{\mathrm{c}}$ & $0.344 \pm 0.016^{\mathrm{b}}$ \\
\hline PSY & $32.31 \pm 1.16^{\mathrm{c}}$ & $2.14 \pm 0.08^{\mathrm{d}}$ & $33.91 \pm 4.69^{c}$ & $4.58 \pm 0.51^{\mathrm{a}}$ & $0.248 \pm 0.002^{\mathrm{d}}$ \\
\hline PGS & $35.77 \pm 1.12^{\mathrm{b}}$ & $2.77 \pm 0.04^{\mathrm{ab}}$ & $73.13 \pm 2.60^{\mathrm{d}}$ & $3.42 \pm 0.03^{b c}$ & $0.213 \pm 0.002^{\mathrm{e}}$ \\
\hline CWSS & $33.35 \pm 0.69^{\mathrm{ac}}$ & $2.55 \pm 0.10^{\mathrm{b}}$ & $59.06 \pm 6.24^{\mathrm{e}}$ & $3.90 \pm 0.14^{\mathrm{ab}}$ & $0.193 \pm 0.003^{\mathrm{e}}$ \\
\hline
\end{tabular}

${ }^{a}$ Rice and MC crackers were sheeted to $2.6 \mathrm{~mm}$, and the other crackers were sheeted to $1.6 \mathrm{~mm}$. Data are shown as mean \pm standard deviation. The different letters associated with the values in the same column indicate significant differences $(p<0.05)$. 


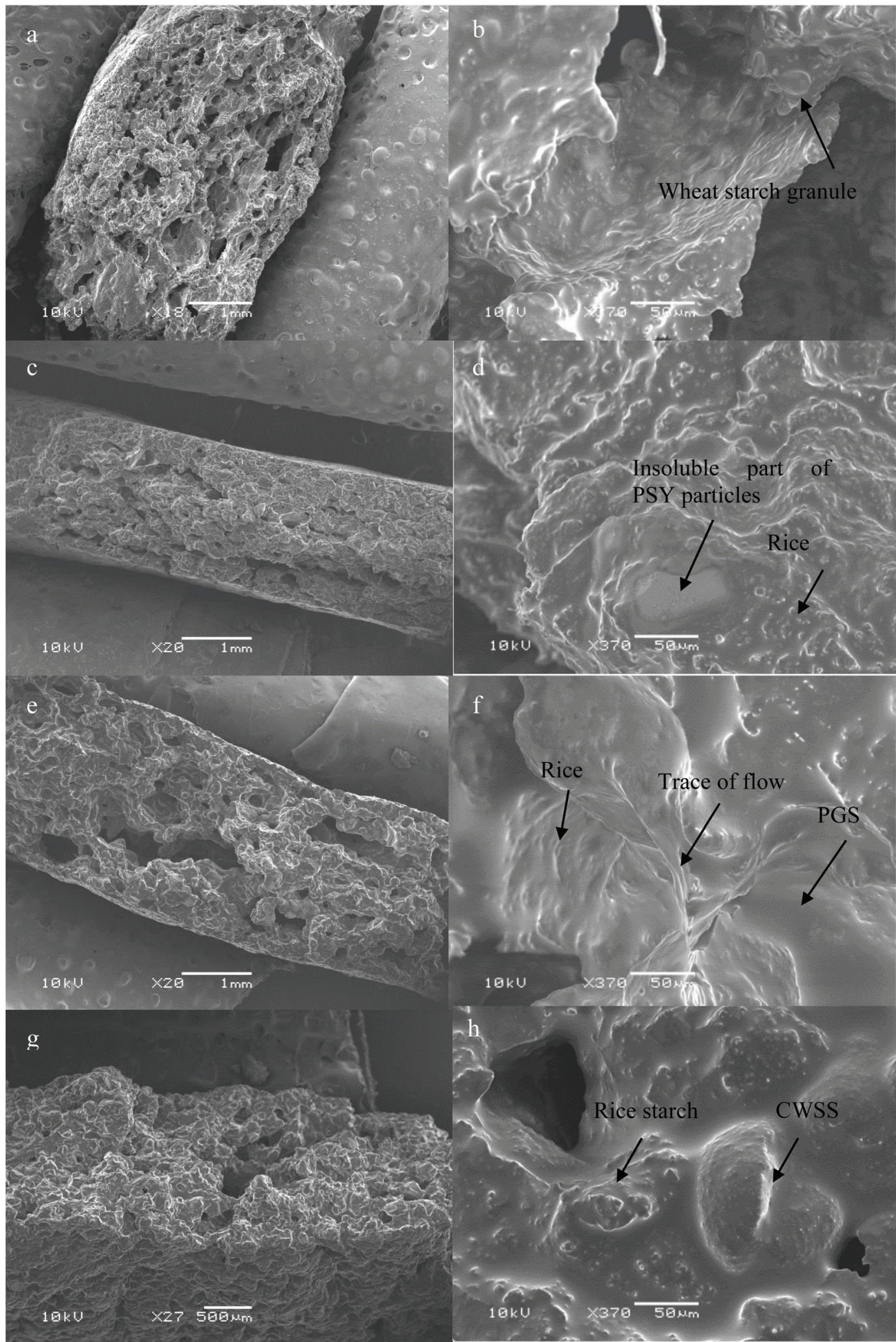

Fig. 5 Scanning electron microscopy images of the wheat crackers ( $a$ and b), PSY crackers (c and d), PGS crackers (e and f) and CWSS crackers (g and h). 


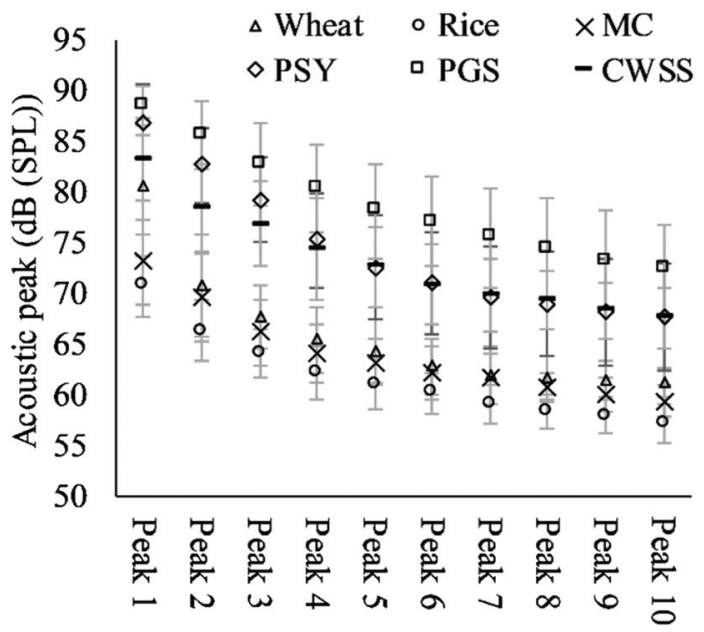

Fig. 6 The highest ten acoustic peaks of the sound pressure levels of the wheat and gluten free crackers. The acoustic peaks from peak 1 to peak 10 are shown from the highest to the lowest values from left to right.

tinuous matrix of the PSY crackers, therefore, predominantly consisted of the hydratable and gel-like part of PSY, which is highly branched heteroxylan. As for the PGS crackers (Fig. 6e and $\mathrm{f}$ ), there were fewer rice starch granules in the matrix as $20 \%$ of rice flour was replaced by PGS, although some starch fragments and aggregates from PGS were observed. In addition to the observation that the PGS doughs were more fluid-like (Fig. 4), it was also flowable during baking as the trace of the extensional flow was observed. The flowability of the PGS doughs during baking allowed the formation and increase of gas cells, which contributed to the highest thickness increase as shown in Table 2. Nevertheless, some large swollen starch granules were observed in the CWSS crackers in addition to the rice starch, which is consistent with what is shown in Fig. 1j.

\subsection{Textural properties and acoustic emission}

The textural properties of the crackers were evaluated and the results are shown in Table 3 . The wheat crackers showed the highest hardness with a relatively high fracturability. As for the gluten free crackers, the PSY crackers had hardness and fracturability closest to those of the wheat crackers, followed by the PGS crackers and CWSS crackers. The rice crackers and MC crackers, however, had very low hardness but high fracturability. It has been hypothesised that the interactions between the carbohydrate polymers at a low moisture level allow the formation of a crystalline-like zone which is critical to hardness and perception of the crispiness of snack products. ${ }^{49}$ However, the texture of the wheat cracker is highly controlled by gluten, ${ }^{50}$ while starch exists as intact granules embedded in the matrix of the gluten network and other ingredients as shown in Fig. 5. The high fracturability of the wheat crackers could be assigned to their higher thickness and flaky structure (Fig. 5a and b). The flaky layers were bent and broken gradually, which contributed to the initial force increase before the first peak, during which the distance indicated fracturability. The force increase with the gradual deformation and collapse of the walls of the gas cells has been observed in cellular products like bread. ${ }^{51}$ However, a hard matrix was absent in the rice crackers, therefore they had the lowest hardness. The high fracturability of the rice crackers is also due to their weak structure. The gradual collapse of the structure caused the force to increase before the first decrease (fracturability). It could be speculated that the high hardness and relatively high fracturability of the PSY crackers are attributed to the selfinteraction between the PSY polymers and interactions with other ingredients. Similarly, a matrix was formed by PGS, which played a similar role to the matrix or film of (pre)gelatinised starch in extruded cereal snacks. ${ }^{52}$ However, the interaction between CWSS was limited to the level of that between granules because the integrity of starch granules was reserved. Hence, CWSS was less efficient than PGS in the formation of the continuous matrix. The low hardness and high fracturability of the MC crackers, which were similar to the rice crackers, indicate that the functionality of MC was limited possibly due to the low moisture level. The molecular mobility of MC was limited at the low water addition level, which inhibited the self-interaction between the MC molecules and the formation of a matrix to bind other ingredients.

When dry food is deformed under an applied force, strain energy is stored. Structural destruction occurs after a critical point is reached, which causes vibration and generates sound pressure waves, during which the stored energy is released as acoustic energy. The sound emission is correlated to the perception of crispiness or crunchiness. ${ }^{53,54}$ The acoustic emis-

Table 3 Textural properties and acoustic emission of the crackers

\begin{tabular}{|c|c|c|c|c|c|}
\hline & Hardness (g) & $\begin{array}{l}\text { Fracturability } \\
(\mathrm{mm})\end{array}$ & $\begin{array}{l}\text { Maximum sound } \\
\text { peak }(\mathrm{dB}(\mathrm{SPL}))\end{array}$ & $\begin{array}{l}\text { Average peak } \\
\text { decrease }(\mathrm{dB}(\mathrm{SPL}))\end{array}$ & $\begin{array}{l}\text { Number of sound } \\
\text { peaks }(\mathrm{dB}(\mathrm{SPL}))\end{array}$ \\
\hline Wheat crackers & $1005 \pm 135^{\mathrm{d}}$ & $0.634 \pm 0.088^{\mathrm{ab}}$ & $80.65 \pm 4.95^{b}$ & $20.74 \pm 5.90^{\mathrm{b}}$ & $8.81 \pm 3.75^{\mathrm{a}}$ \\
\hline Rice crackers $^{a}$ & $150 \pm 26^{\mathrm{a}}$ & $0.748 \pm 0.204^{\mathrm{a}}$ & $70.72 \pm 3.05^{\mathrm{a}}$ & $15.55 \pm 2.70^{\mathrm{a}}$ & $11.57 \pm 3.08^{\mathrm{ab}}$ \\
\hline 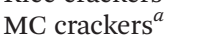 & $326 \pm 50^{\mathrm{b}}$ & $0.727 \pm 0.133^{\mathrm{a}}$ & $73.12 \pm 4.13^{\mathrm{a}}$ & $18.69 \pm 5.58^{\mathrm{ab}}$ & $11.88 \pm 4.87^{\mathrm{ab}}$ \\
\hline PSY crackers & $801 \pm 166^{c}$ & $0.568 \pm 0.117^{b}$ & $86.87 \pm 3.73^{c}$ & $19.78 \pm 5.51^{\mathrm{ab}}$ & $15.27 \pm 5.18^{\mathrm{b}}$ \\
\hline PGS crackers & $667 \pm 129^{c}$ & $0.330 \pm 0.087^{\mathrm{c}}$ & $88.41 \pm 1.86^{c}$ & $23.27 \pm 3.28^{\mathrm{b}}$ & $30.31 \pm 11.29^{c}$ \\
\hline CWSS crackers & $461 \pm 163^{b}$ & $0.329 \pm 0.129^{c}$ & $83.20 \pm 4.06^{\mathrm{b}}$ & $23.08 \pm 4.60^{\mathrm{b}}$ & $22.00 \pm 8.49^{\mathrm{d}}$ \\
\hline
\end{tabular}

${ }^{a}$ Rice and MC crackers were sheeted to $2.6 \mathrm{~mm}$, and the other crackers were sheeted to $1.6 \mathrm{~mm}$. Data are shown as mean \pm standard deviation. The different letters associated with the values in the same column indicate significant differences $(p<0.05)$. 
sion during the three-point bending tests was recorded, and the maximum sound peak, average peak decrease and number of sound peaks are shown in Table 3. The first ten acoustic peaks recorded for each cracker were ordered from high to low and are shown in Fig. 6. As shown in Table 3, the rice crackers and MC crackers had lower maximum sound peaks and average peak decreases compared to the wheat crackers. The observation is consistent with hardness. However, the addition of CWSS, PSY, and PGS in the crackers significantly increased the maximum sound peak and average peak decrease compared to the wheat crackers, which is also shown in Fig. 6, suggesting that the highest ten acoustic peaks of the PGS, CWSS, and PSY crackers were generally higher than those of the wheat, rice, and MC crackers. The observations indicate that higher levels of sound were emitted during the breaking of these three types of crackers. The gluten free crackers also had a higher number of sound peaks than the wheat crackers, which was opposite to hardness. During each structural destruction, there is a force decrease accompanied by an acoustic event. ${ }^{53,55}$ Saeleaw et al. ${ }^{56}$ observed the decrease of hardness with a lower number of sound peaks due to the increase in the pores in the products. Therefore, the high maximum sound peak accompanied by the high hardness of the wheat crackers is assigned to the gluten network, while the low number of sound peaks indicates that there might be weak points in the flaky structure which do not contribute to the strength of the structure and sound emission. In contrast, the gluten free crackers did not show a flaky structure. The breaking and sound emission during tests were caused by multiple fractures across the whole cracker due to inhomogeneity and weak points instead of predominantly at the contacting position with the probe. It is also noticed from Fig. 6 that compared to the wheat crackers, there was less difference between the highest acoustic peak and the second highest peak of all gluten free crackers, which indicates that the break and release of the acoustic energy were less sharp than those of the wheat crackers. It also evidences the multiple fractures of the gluten free crackers.

\section{Conclusion}

The production of gluten free crackers was developed by the incorporation of PSY, MC, PGS, and CWSS and compared to wheat crackers. PSY and PGS form matrices which hold flour particles together and form cohesive doughs. The PSY and PGS matrices in the crackers contribute to comparable hardness and sound release to wheat crackers during breaking. Multiple fractures occur across gluten free crackers during breaking, while there is a shape break of wheat crackers due to their laminar structure. PSY increases the moisture content and lowers the water activity of gluten free crackers due to its high water retention ability. PGS significantly increases the shapeability of gluten free doughs and allows them to flow during baking. MC increases the viscosity and leads to significant effects on the swelling and breakdown behaviours of starch granules which dramatically modify the pasting profile. However, the low water addition level of crackers inhibits selfinteraction of MC molecules and limits their functionality; therefore MC crackers have a poorer quality similar to rice crackers. CWSS crackers also show certain levels of hardness, fracturability, and sound release comparable to wheat crackers but the interaction is mainly between their starch granules which limit their ability to form a strong matrix. Therefore, further studies will be based on PSY and PGS and focus on the modification of the process.

\section{Conflicts of interest}

Bruce R. Linter is employed by PepsiCo, Inc. The views and opinions expressed in this manuscript are those of the authors and do not necessarily reflect the position or policy of PepsiCo, Inc.

\section{Acknowledgements}

This work was supported by the University of Nottingham (Vice-Chancellor's Scholarship for Research Excellence (International)) and PepsiCo, Inc. The authors would like to thank Khatija Nawaz Husain for her technical help with confocal laser scanning microscopy, which was conducted at the School of Life Sciences Imaging and Microscopy Facility (SLIM) at the University of Nottingham. Scanning electron microscopy images were collected at the Nanoscale and Microscale Research Centre (nmRC) at the University of Nottingham.

\section{Notes and references}

1 D. Manley, Classification of biscuits, in Manley's Technology of Biscuits, Crackers and Cookies, ed. D. Manley, Woodhead Publishing, Cambridge, 2011, pp. 271-278.

2 D. Manley, Classification based on enrichment of the formulation, in Technology of biscuits, crackers and cookies, ed. D. Manley, Woodhead Publishing, Cambridge, 3rd edn, 2000.

3 D. Manley, Cream crackers, in Manley's Technology of Biscuits, Crackers and Cookies, ed. D. Manley, Woodhead Publishing, Cambridge, 4th edn, 2011, pp. 279-292.

4 A. Fasano and C. Catassi, Current approaches to diagnosis and treatment of celiac disease: An evolving spectrum, Gastroenterology, 2001, 120, 636-651.

5 S. J. Shepherd and P. R. Gibson, Nutritional inadequacies of the gluten-free diet in both recently-diagnosed and longterm patients with coeliac disease, J. Hum. Nutr. Diet., 2013, 26, 349-358.

6 I. Sedej, M. Sakač, A. Mandić, A. Mišan, M. Pestorić, O. Šimurina and J. Čanadanović-Brunet, Quality assessment of gluten-free crackers based on buckwheat flour, LWT - Food Sci. Technol., 2011, 44, 694-699. 
7 O. Radočaj, E. Dimić and R. Tsao, Effects of hemp (Cannabis sativa L.) seed oil press-cake and decaffeinated green tea leaves (Camellia sinensis) on functional characteristics of gluten-free crackers, J. Food Sci., 2014, 79, C318C325.

8 J. Han, J. A. M. Janz and M. Gerlat, Development of glutenfree cracker snacks using pulse flours and fractions, Food Res. Int., 2010, 43, 627-633.

9 A. Haque and E. R. Morris, Combined use of ispaghula and HPMC to replace or augment gluten in breadmaking, Food Res. Int., 1994, 27, 379-393.

10 A. Lazaridou, D. Duta, M. Papageorgiou, N. Belc and C. G. Biliaderis, Effects of hydrocolloids on dough rheology and bread quality parameters in gluten-free formulations, J. Food Eng., 2007, 79, 1033-1047.

11 K. D. Nishita, R. L. Roberts, M. M. Bean and B. M. Kennedy, Development of a yeast-leavened rice-bread formula, Cereal Chem., 1976, 53, 626-635.

12 Y. Ren, B. R. Linter, R. Linforth and T. J. Foster, A comprehensive investigation of gluten free bread dough rheology, proving and baking performance and bread qualities by response surface design and principal component analysis, Food Funct., 2020, 11, 5333-5345.

13 D. Sabanis, D. Lebesi and C. Tzia, Effect of dietary fibre enrichment on selected properties of gluten-free bread, LWT - Food Sci. Technol., 2009, 42, 1380-1389.

14 R. Moreira, F. Chenlo and M. D. Torres, Effect of chia (Sativa hispanica L.) and hydrocolloids on the rheology of gluten-free doughs based on chestnut flour, LWT - Food Sci. Technol., 2013, 50, 160-166.

15 M. Lauková, Z. Kohajdová and J. Karovicová, Effect of incorporating potato dietary fibre to wheat dough on the quality of baked rolls, Acta Chim. Slovaca, 2016, 9, 14-18.

16 J. P. Karl and E. Saltzman, The role of whole grains in body weight regulation, Adv. Nutr., 2012, 3, 697-707.

17 Y. Tousen, M. Uehara, F. Abe, Y. Kimira and Y. Ishimi, Effects of short-term fructooligosaccharide intake on equol production in Japanese postmenopausal women consuming soy isoflavone supplements: a pilot study, Nutr. J., 2013, 12, 127.

18 L. M. Steffen, D. R. Jacobs Jr., J. Stevens, E. Shahar, T. Carithers and A. R. Folsom, Associations of whole-grain, refined-grain, and fruit and vegetable consumption with risks of all-cause mortality and incident coronary artery disease and ischemic stroke: the Atherosclerosis Risk in Communities (ARIC) Study, Am. J. Clin. Nutr., 2003, 78, 383-390.

19 R. Rodríguez, A. Jiménez, J. Fernández-Bolaños, R. Guillén and A. Heredia, Dietary fibre from vegetable products as source of functional ingredients, Trends Food Sci. Technol., 2006, 17, 3-15.

20 F. M. Silva, C. K. Kramer, J. C. de Almeida, T. Steemburgo, J. L. Gross and M. J. Azevedo, Fiber intake and glycemic control in patients with type 2 diabetes mellitus: a systematic review with meta-analysis of randomized controlled trials, Nutr. Rev., 2013, 71, 790-801.
21 J. N. BeMiller and K. C. Huber, Physical modification of food starch functionalities, Annu. Rev. Food Sci. Technol., 2015, 6, 19-69.

22 M. Z. I. Sarker, M. A. Elgadir, S. Ferdosh, M. J. H. Akanda, P. Aditiawati and T. Noda, Rheological behavior of starchbased biopolymer mixtures in selected processed foods, Starch/Staerke, 2013, 65, 73-81.

23 S. Rajagopalan and P. A. Seib, Granular cold-water-soluble starches prepared at atmospheric pressure, J. Cereal Sci., 1992, 16, 13-28.

24 L. A. Bello-Pérez, R. Romero-Manilla and O. Paredes-López, Preparation and properties of physically modified banana starch prepared by alcoholic-alkaline treatment, Starch/ Staerke, 2000, 52, 154-159.

25 B. Zhang, S. Dhital, E. Haque and M. J. Gidley, Preparation and characterization of gelatinized granular starches from aqueous ethanol treatments, Carbohydr. Polym., 2012, 90, 1587-1594.

26 J. Jane, S. A. S. Craig, P. A. Seib and R. C. Hoseney, Characterization of granular cold water-soluble starch, Starch/Staerke, 1986, 38, 258-263.

27 G. Bortnowska, J. Balejko, G. Tokarczyk, A. RomanowskaOsuch and N. Krzemińska, Effects of pregelatinized waxy maize starch on the physicochemical properties and stability of model low-fat oil-in-water food emulsions, Food Hydrocolloids, 2014, 36, 229-237.

28 J.-l. Jane, Preparation and food applications of physically modified starches, Trends Food Sci. Technol., 1992, 3, 145148.

29 Y. Ren, B. R. Linter and T. J. Foster, Starch replacement in gluten free bread by cellulose and fibrillated cellulose, Food Hydrocolloids, 2020, 107, 105957.

30 S. Meiboom and D. Gill, Modified Spin-Echo method for measuring nuclear relaxation times, Rev. Sci. Instrum., 1958, 29, 688-691.

31 J. Delcour and R. Hoseney, Principles of cereal science and technology, AACC International, Saint Paul, 3rd edn, 2010.

32 P. Y. Chiang and A. I. Yeh, Effect of soaking on wet-milling of rice, J. Cereal Sci., 2002, 35, 85-94.

33 M. Mariotti, M. Lucisano, M. A. Pagani and P. K. W. Ng, The role of corn starch, amaranth flour, pea isolate, and psyllium flour on the rheological properties and the ultrastructure of gluten-free doughs, Food Res. Int., 2009, 42, 963-975.

34 Y. Ren, G. E. Yakubov, B. R. Linter, W. MacNaughtan and T. J. Foster, Temperature fractionation, physicochemical and rheological analysis of psyllium seed husk heteroxylan, Food Hydrocolloids, 2020, 104, 105737.

35 W. Burchard, Solubility and solution structure of cellulose derivatives, Cellulose, 2003, 10, 213-225.

36 The Dow Chemical Company, Product Safety Assessment METHOCEL ${ }^{\mathrm{TM}}$ Cellulose Ethers, 2013.

37 A. Anastasiades, S. Thanou, D. Loulis, A. Stapatoris and T. D. Karapantsios, Rheological and physical characterization of pregelatinized maize starches, J. Food Eng., 2002, 52, 57-66. 
38 D. M. Dries, S. V. Gomand, B. Goderis and J. A. Delcour, Structural and thermal transitions during the conversion from native to granular cold-water swelling maize starch, Carbohydr. Polym., 2014, 114, 196-205.

39 S. Naruenartwongsakul, M. S. Chinnan, S. Bhumiratana and T. Yoovidhya, Pasting characteristics of wheat flourbased batters containing cellulose ethers, LWT - Food Sci. Technol., 2004, 37, 489-495.

40 A. Sullo and T. J. Foster, Characterisation of starch/cellulose blends, Annu. Trans. - Nord. Rheol. Soc., 2010, 18, 17.

41 I. M. Krieger and T. J. Dougherty, A mechanism for nonnewtonian flow in suspensions of rigid spheres, Trans. Soc. Rheol., 1959, 3, 137-152.

42 M. R. Debet and M. J. Gidley, Why do gelatinized starch granules not dissolve completely? Roles for amylose, protein, and lipid in granule "ghost" integrity, J. Agric. Food Chem., 2007, 55, 4752-4760.

43 K. N. Nakorn, T. Tongdang and P. Sirivongpaisal, Crystallinity and Rheological Properties of Pregelatinized Rice Starches Differing in Amylose Content, Starch/Staerke, 2009, 61, 101-108.

44 T. Amari, Non-linear viscoelastic properties of concentrated suspensions, Prog. Org. Coat., 1997, 31, 11-19.

45 B. S. Khatkar and J. D. Schofield, Dynamic rheology of wheat flour dough. I. Non-linear viscoelastic behaviour, J. Sci. Food Agric., 2002, 82, 827-829.

46 L. Slade, H. Levine and J. Finley, Protein-water interactions: Water as a plasticizer of gluten and other protein polymers, in Protein quality and the effects of processing, ed. R. D. Phillips and J. W. Finley, Marcel Dekker, New York, 1989, pp. 9-124.
47 M. Kaur, K. S. Sandhu, A. Arora and A. Sharma, Gluten free biscuits prepared from buckwheat flour by incorporation of various gums: Physicochemical and sensory properties, LWT - Food Sci. Technol., 2015, 62, 628-632.

48 K. Kulp, M. Olewnik, K. Lorenz and F. Collins, Starch functionality in cookie systems, Starch/Staerke, 1991, 43, 53-57.

49 E. E. Katz and T. P. Labuza, Effect of water activity on the sensory crispness and mechanical deformation of snack food products, J. Food Sci., 1981, 46, 403-409.

50 A. Nikolaidis and T. P. Labuza, Glass transition state diagram of a baked cracker and its relationship to gluten, J. Food Sci., 1996, 61, 803-806.

51 A. E. Baker, C. E. Walker and K. Kemp, An optimum compression depth for measuring bread crumb firmness, Cereal Chem., 1988, 65, 302-307.

52 Y. Gat and L. Ananthanarayan, Effect of extrusion process parameters and pregelatinized rice flour on physicochemical properties of ready-to-eat expanded snacks, J. Food Sci. Technol., 2015, 52, 2634-2645.

53 J. Chen, C. Karlsson and M. Povey, Acoustic envelope detector for crispness assessment of biscuits, J. Texture Stud., 2005, 36, 139-156.

54 B. K. Drake, Food crushing sounds: Comparisons of objective and subjective data, J. Food Sci., 1965, 30, 556-559.

55 P. Varela, A. Salvador and S. M. Fiszman, Methodological developments in crispness assessment: Effects of cooking method on the crispness of crusted foods, LWT - Food Sci. Technol., 2008, 41, 1252-1259.

56 M. Saeleaw, K. Dürrschmid and G. Schleining, The effect of extrusion conditions on mechanical-sound and sensory evaluation of rye expanded snack, J. Food Eng., 2012, 110, 532-540. 